\title{
THE ABSENCE OF FLARES IN $\lambda 3835$ AND THE HEATING OF THE CHROMOSPHERE
}

\author{
HAROLD ZIRIN \\ Big Bear Solar Observatory, Hale Observatories, California Institute of Technology, \\ Carnegie Institution of Washington
}

(Received 5 April, 1972)

\begin{abstract}
Simultaneous observations of flares in $\mathrm{H} \alpha$ and a band $15 \AA$ wide centered on $3835 \AA$ show no change whatever in $3835 \AA$ at the time of several flares, although the chromospheric network is easily visible. Flares are therefore transparent in this wavelength.

Since the flare represents a test heat input from above, and no brightening of the network appears, we conclude it is impossible for the corona to produce the bright network by downward convection.
\end{abstract}

In August 1970 a Chapman (1970a)-type broad band filter at $3835 \AA$ was operated at Big Bear for a number of days. Films were obtained with resolution of one arc second. Observations of the chromospheric network and Ellerman bombs with the filter have been given by Vorpahl and Pope (1972). The purpose of this note is to relate a negative result on flares.

In the period of observation, significant flares were only recorded on August 3, when five flares were observed in the leading part of McMath 11457. Three of these flares occurred while observations were in progress in both $\mathrm{H} \alpha$ and $3835 \AA$ at 1857 , 1913 and 1928 UT. These flares did not produce reported microwave emission, but type III bursts were reported. The $\mathrm{H} \alpha$ flares were of class 1 , and occurred in a region of inverted polarity just ahead of the sunspot.

Figure 1 shows simultaneous pictures of one flare in 3835 and $\mathrm{H} \alpha$. Although the chromospheric network is easily visible in 3835 there is no evidence for flare emission at all. Moreover in cine projection no change of brightness is apparent at the time of the flare except for the chromospheric oscillation normally visible in 3835. Flares recurred in the region during the next hour, again with no visible emission in 3835 (Figure 2).

The significance on this observation is to delineate the solar flare as a purely high chromospheric phenomenon. If the flare has any heating effect on the temperature in the network region, it can be expected to produce an increase in emission from the network, which we see in this wavelength. We have seen such effects in the $\mathrm{K}$ line, where flares produce brightening of the associated network. Furthermore, the events observed were at E75, so the height to which we see is somewhat above that at which the network may be seen in the disk center. Using the BCA atmosphere and Chapman's (1970b) estimate that through the 3835 filter we reach $\tau=1$ where $\tau=0.2-0.3$ at $\lambda 5000$, then for $\theta=75^{\circ}$ we look at $\tau \approx 0.05$ in $\lambda 5000$, or a density of $1.2 \times 10^{-7} \mathrm{gm} \mathrm{cm}^{-3}$.

The region covered by the 3835 filter is a deep depression in the continuum produced by the confluence of $\mathrm{CN}$ bands and very strong neutral $\mathrm{Fe}$ and $\mathrm{Mg}$ lines. As Chapman 
a

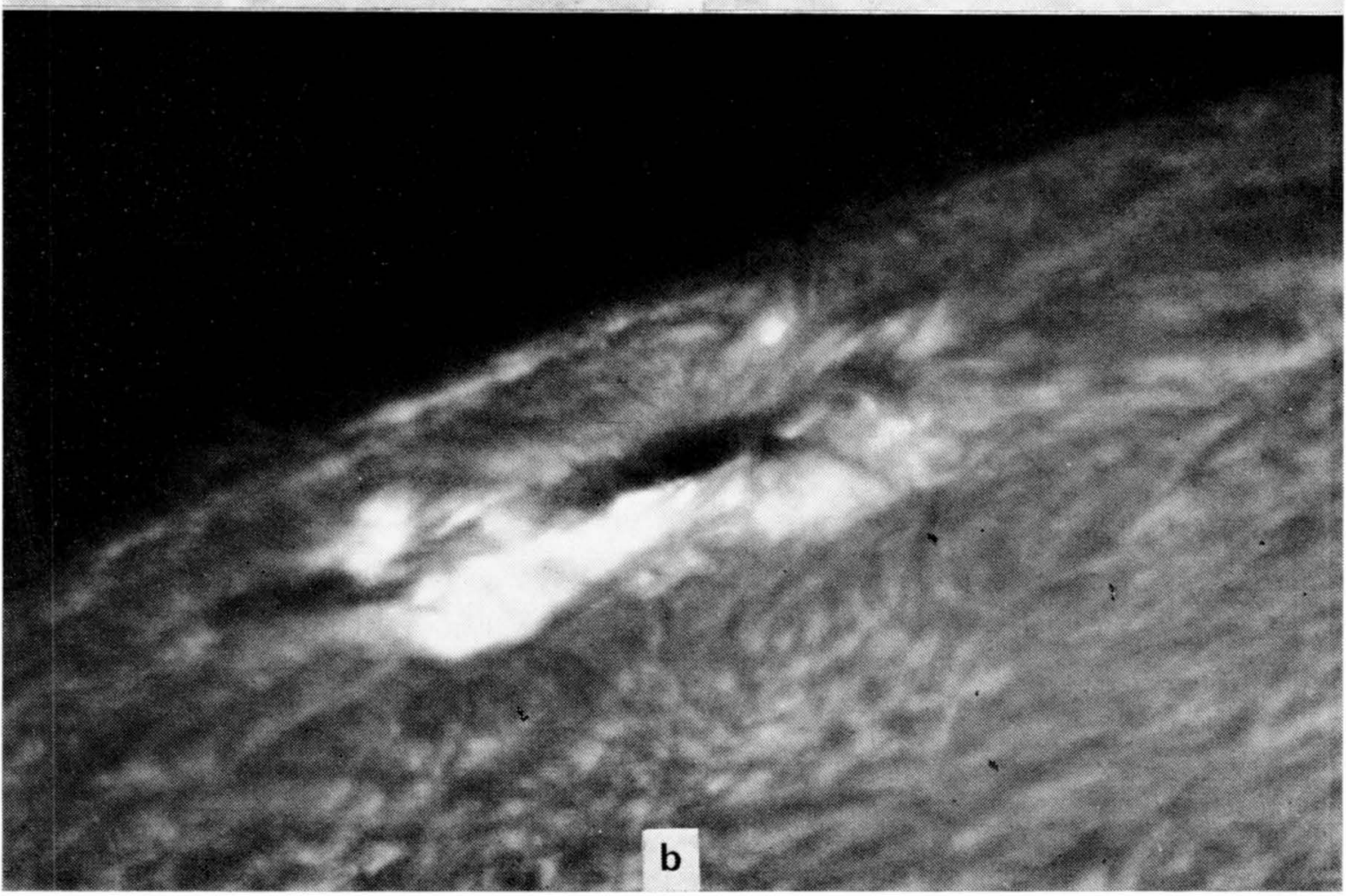

Fig. 1. Flare at $1856 \mathrm{UT}$, August 3, 1971.

$\begin{array}{ll}\text { a. } 3835 \AA & \text { b. } \mathrm{H} \alpha\end{array}$

Note that there is no brightening in $3835 \AA$. 


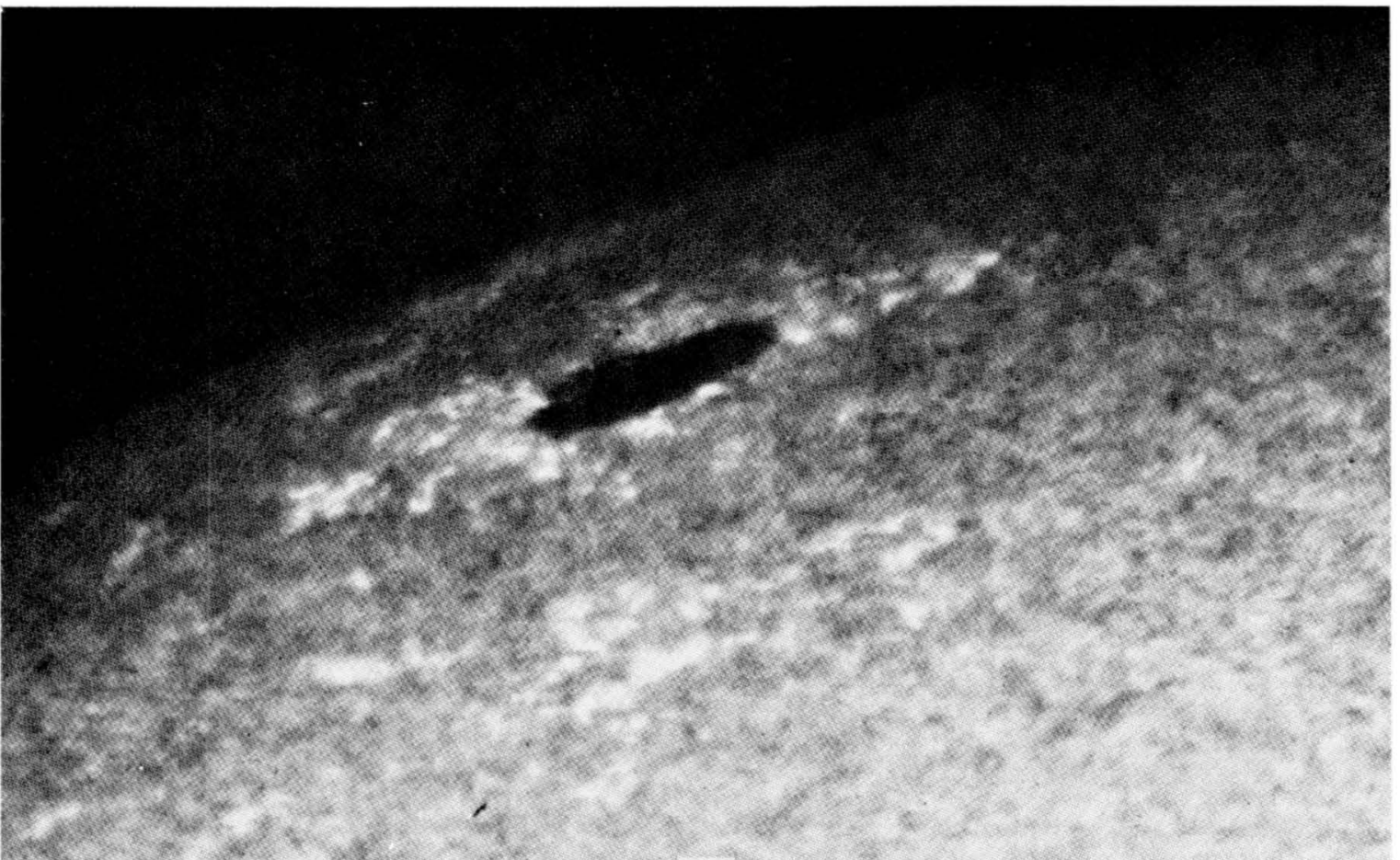

a

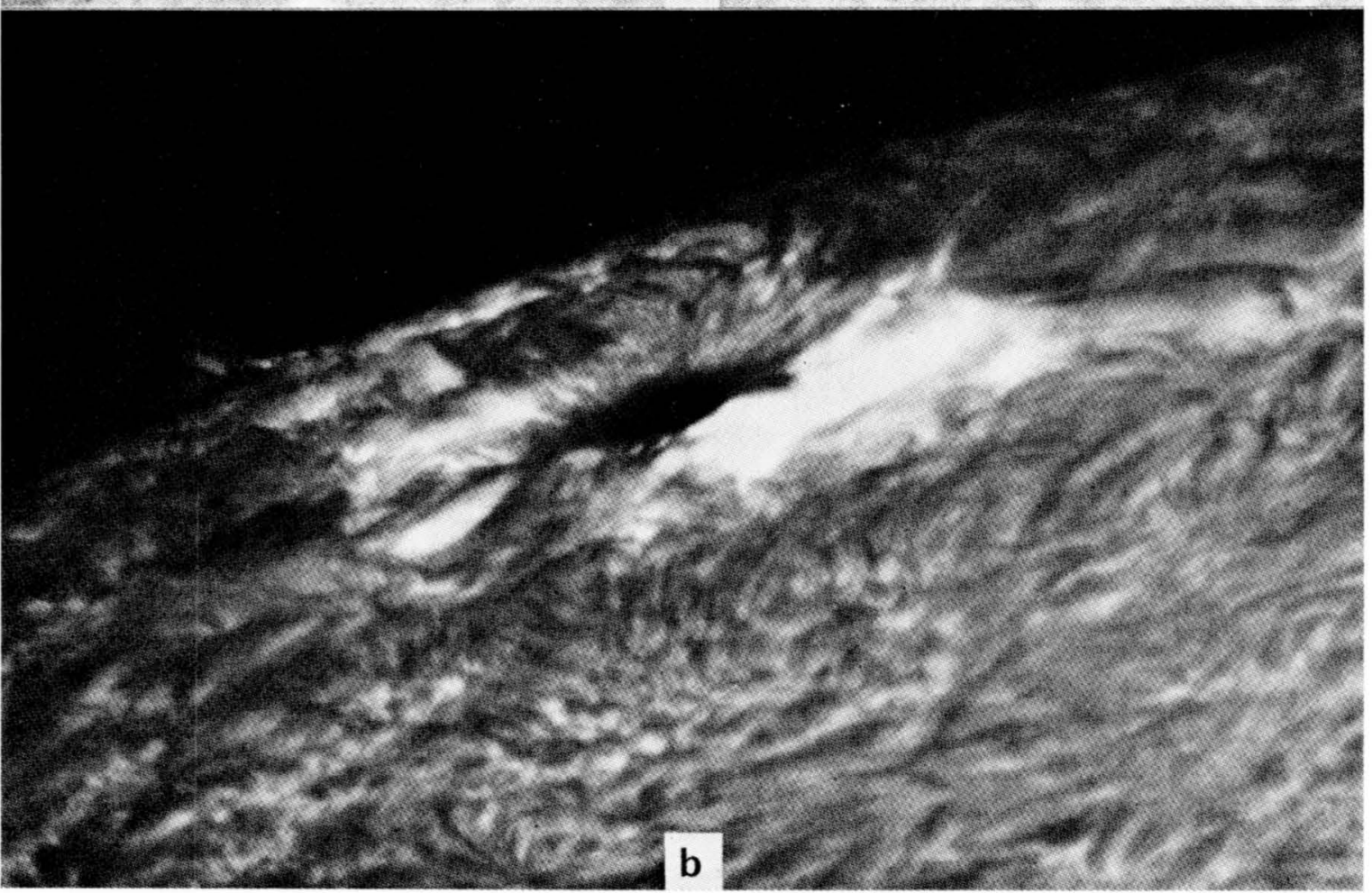

Fig. 2. Flare at 1927 UT, August 3, 1971.
a. $3835 \AA$
b. $\mathrm{H} \alpha$

Note that there has been no change from Figure 1 in $3835 \AA$. 
points out these lines are weakened in regions of higher field, presumably because the photosphere is heated there, so a bright network is seen. The lack of flare brightening implies that the flare does not produce any appreciable heating of the photosphere. If we use the BCA temperature at this depth, $4920 \mathrm{~K}$, we find that a $2 \%$ increase in brightness is produced by a $12^{\circ}$ temperature increase. Such an increase would be detectable on our films, which have a gamma of four. The thermal energy involved to raise a typical plagette to this temperature is about $10^{26} \mathrm{erg}$, easily available in the flare. Apparently it is radiated away before it reaches the surface.

Dr Chapman kindly informed me that a low resolution patrol was operated at Hawaii for some time with negative results except for one large class $2 \mathrm{~B}$ flare and two undocumented flares. It would appear that in the large flares enough heat reaches the surface. Kuperus and Athay (1967) have proposed a model in which spicules are produced by conduction downward from the corona to the upper chromosphere. I have already pointed out (Zirin, 1971) that the existence of chromospheres in stars with gravity much too low to hold coronas ruled out the back-conduction model. In this case the sun performs an experiment to test the effect on the low chromosphere of a certain heat input, namely a flare. The fact that there is no change in the network shows that that brightening does not come from above. Of course this does not exclude an important role for back-conduction at higher levels, as in the Kuperus-Athay model.

Because of the complexity of the lines filling the range of the filter, we can learn little about flares from this observation, except the fact that they clearly occur above the height in question, namely $170 \mathrm{~km}$ above the zero level, or, more accurately, above $\tau=0.05$ in the continuum at $5000 \mathrm{~K}$.

\section{Acknowledgements}

I am indebted to Tom Pope for getting the observations and Dr Gary Chapman for several conversations.

This work was supported by NASA under grant NGR 05002034 and NSF under grant Ga. 24015.

Note added in proof: We observed brightening in $3835 \AA$ in two great flares Aug. 2 , 1972; in the first, an impulsive flare at 1839 produced several short-lived $(\sim 10 \mathrm{~s})$ bright points along the neutral line; in the second, a large, slower flare produced small gradual brightening along the moving edge of one strand $(\sim 2150 \mathrm{UT})$.

\section{References}

Chapman, G.: 1970a, Solar Phys. 13, 78.

Chapman, G.: 1970b, Solar Phys. 14, 315.

Kuperus, M. and Athay, R. G.: 1967, Solar Phys. 1, 361.

Zirin, H.: 1971, Phil. Trans. Roy. Soc. London A, 270, 183.

Vorpahl, J. and Pope, T.: 1972, Solar Phys. 25, 347. 